\title{
ISSUES ON REPRODUCIBILITY/RELIABILITY OF MAGNETIC NDE METHODS
}

\author{
M. Namkung \\ NASA Langley Research Center \\ Hampton, VA 23681 \\ J. P. Fulton, B. Wincheski, and S. Nath \\ Analytic Services and Materials, Inc., \\ Hampton, VA 23666
}

\section{INTRODUCTION}

One of the critical elements related to the practicality of any NDE technique is its reproducibility under nominally the same inspection conditions. The results of certain test methodologies, however, are not always repeatable and understanding the origin of the irreproducibility is often as critical as obtaining reproducible results. One example is the characterization of residual stress in structural ferromagnets using the magnetoacoustic (MAC) method [1]. Although it has not been widely publicized, the test results of this method are known to be time-dependent. Two distinct types of time dependencies have been observed during testing. The first type has a clearly definable relaxation time, while no such trend has been observed for the second.

The time dependence associated with the well defined relaxation time, while occuring infrequently, causes the test resultsto change in a very predictable way [2]. This phenomenon is caused by the diffusional motion of interstitial carbon atoms from energetically unfavorable to favored octahedral sites. The change in the domain magnetization orientation alters the preference among the sites causing the carbon atoms to migrate [3]. Even though the variation in the test results over time is large, the time dependence is not a critical problem since the trend is predictable. The real problem is the second type of time dependence which has been observed in virtually all the steel samples tested in our laboratory during tests to determine the applied stress dependence of the MAC curves. The variation in the test results is much smaller compared to the diffusional effects discussed above, but it is still large enough to significantly affect laboratory test results used as calibration data.

The purpose of the present study is to systematically investigate the time dependence of the second type, to find out the range and, if possible, the origin of the variation in the test results. For this, MAC curves were obtained under various stress levels and the tests were repeated over time. Particular attention was given to whether noise in the measuring device or a change in the laboratory environment could have been a contributing factor. 


\section{EXPERIMENTS}

The steel samples used for the study were cut from C- and U-class railroad wheels. The MAC behavior of these samples was reported previously [4]. Each steel sample was first machined to be a cylindrical rod of $3.175 \mathrm{~cm}(1.25 \mathrm{in})$ in diameter and $26.67 \mathrm{~cm}(10.5$ in) in length. The center portion of the samples were further machined to form a pair of flat and parallel surfaces for the launch and reflection of the ultrasonic pulses. Data acquisition involved two major elements; magnetic and acoustic measurements. Throughout the experiment the net magnetic induction, B, was measured by integrating the induction pickup coil output using an integrating fluxmeter. The acoustic measurements employed the phaselocked technique which will be described in the following.

In the phase locked technique a reference sinusoidal signal generated from a voltagecontrolled oscillator is gated to form a series of tone bursts which are fed into the ultrasonic transducer. Short bursts of ultrasonic wavelets travel through the test sample and are then detected by the same transducer (pulse-echo mode). The detected tone bursts are phasecompared against the reference signals where this phase difference can be written as:

$$
\phi=2 \pi \mathrm{fl} / \mathrm{v}
$$

where $f$ is the frequency of the ultrasonic waves, 1 is the total path length and $v$ is the ultrasonic phase velocity. The differential form of the above expression can be easily obtained as

$$
\Delta \phi / \phi=\Delta \mathrm{f} / \mathrm{f}+\Delta \mathrm{l} / \mathrm{l}-\Delta \mathrm{v} / \mathrm{v}
$$

If the phase difference is kept constant, the left hand side of the above expression vanishes. This is called the phase-locked condition and can be achieved in practice by varying the frequency of the ultrasonic waves. Hence, the measured quantity is actually the fractional frequency shift under the phase-locked condition which can be written as

$$
\Delta \mathrm{f}(\mathrm{B}) / \mathrm{f}=\Delta \mathrm{v}(\mathrm{B}) / \mathrm{v}-\Delta \mathrm{l}(\mathrm{B}) / 1
$$

All of the terms in the above expression are functions of B, the magnetic induction, but they can also be functions of other parameters such as the magnetization, $\mathrm{M}$, or the internal magnetic field, $\mathrm{H}$. The first term on the right hand side of the above expression is due to the $\Delta \mathrm{E}$ effect, where $\mathrm{E}$ is the modulus of elasticity, which is mainly caused by the motion of non$180^{\circ}$ domain wall motion [5], and the second term is due to magnetostriction. In the present experiment, both the external magnetic field and the uniaxial stress were applied along the cylindrical axis of the sample. Ultrasonic compressional waves of $5 \mathrm{MHz}$ were propagated perpendicular to the field/stress axis.

There exist three potential factors that can contribute to the poor reproducibility of the MAC test results, namely: changes in the mechanical coupling between the ultrasonic transducer and the sample, drift in the measuring electronic circuits, and changes in ambient temperature. To confirm that a change in coupling is not a factor, the state of the applied stress was altered continuously and abruptly while monitoring $\Delta \mathrm{f}(\mathrm{B}) / \mathrm{f}$. The ambient temperature was monitored using a platinum resistance temperature sensor. The sensors are capable of monitoring temperature changes as small as $0.01^{\circ} \mathrm{C}$.

\section{RESULTS}

Fig. 1 shows $\Delta \mathrm{f}(\sigma) / \mathrm{f}$ curves obtained with the sample cut from a U-class railroad wheel where $\sigma$ is slowly varying uniaxial stress. Twelve different measurements were performed over an 18 hour period. The duration of each load cycle was approximately 80 sec- 


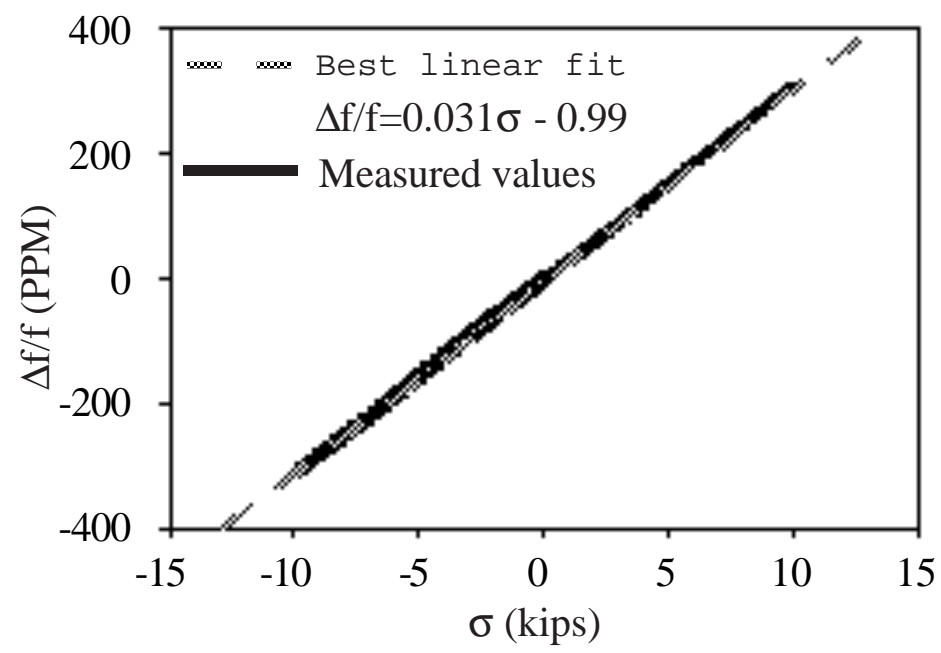

Fig. 1. Results of 12 stress-acoustic measurements with the U-class wheel steel sample made over a period of 18 hours.

onds. The results in Fig. 2 summarize a total of seven measurements performed with a sample cut from a C-class wheel over a period of 3 hours. The results of these two figures confirm two facts: first, the repeated load cycles do not cause any discontinuous variation in the transducer-sample coupling state and, second, continuous changes in microstructure, such as irreversible dislocation motion, do not cause any appreciable contribution to irreproducibility of the acoustic response.

Fig. 3 shows the results of $\Delta \mathrm{f}(\mathrm{B}) / \mathrm{f}$ curves at various stress levels with the U-class sample taken on June 21, 1994. These results can be compared with the results of Fig. 4 obtained for the same sample on June 24, 1994 under nominally identical conditions. Only the curves taken under a -100 and a $-200 \mathrm{MPa}$ load are seen to be slightly different and the irreproducibility of these $\Delta \mathrm{f}(\mathrm{B}) / \mathrm{f}$ curves is minimal. The results obtained with the C-class samples are shown in Fig. 5 and Fig. 6. The trend of irreproducibility in these sample is seen to be more pronounced than that of the U-class samples shown in the previous two figures.

It should be noted that the above results may be affected by changes in the room temperature which can occur during the approximately 20 seconds needed to acquire the data points of each MAC curve. To estimate the effects of fluctuations in room temperature, the long term temperature effects were investigated and the results are shown in Fig. 7. The periodic temperature fluctuations are due to the cyclical operation of an air-conditioner in

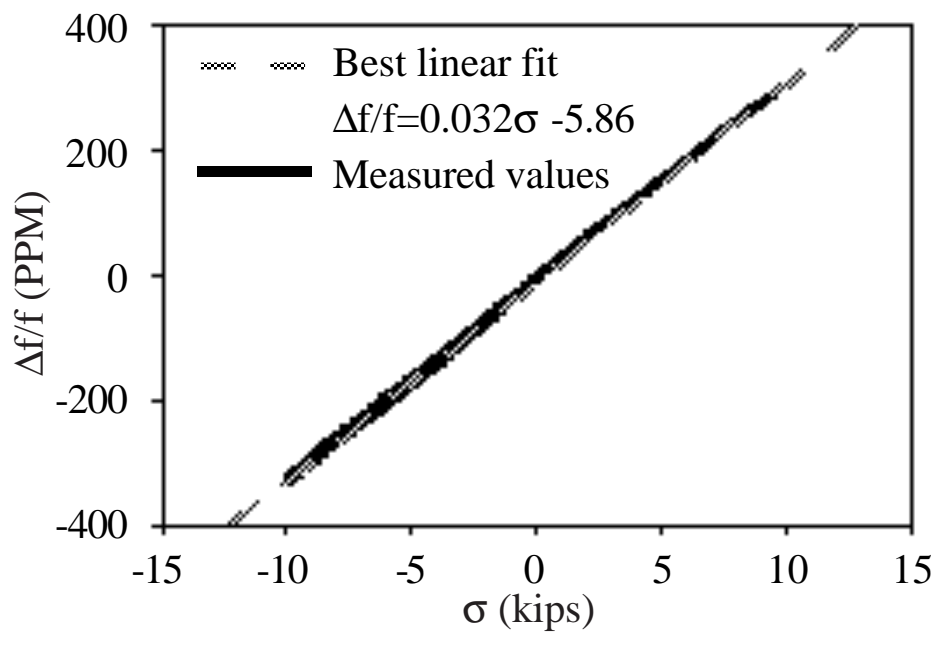

Fig. 2. Results of 7 stress-acoustic measurements with the C-class wheel steel sample made over a period of 3 hours. 


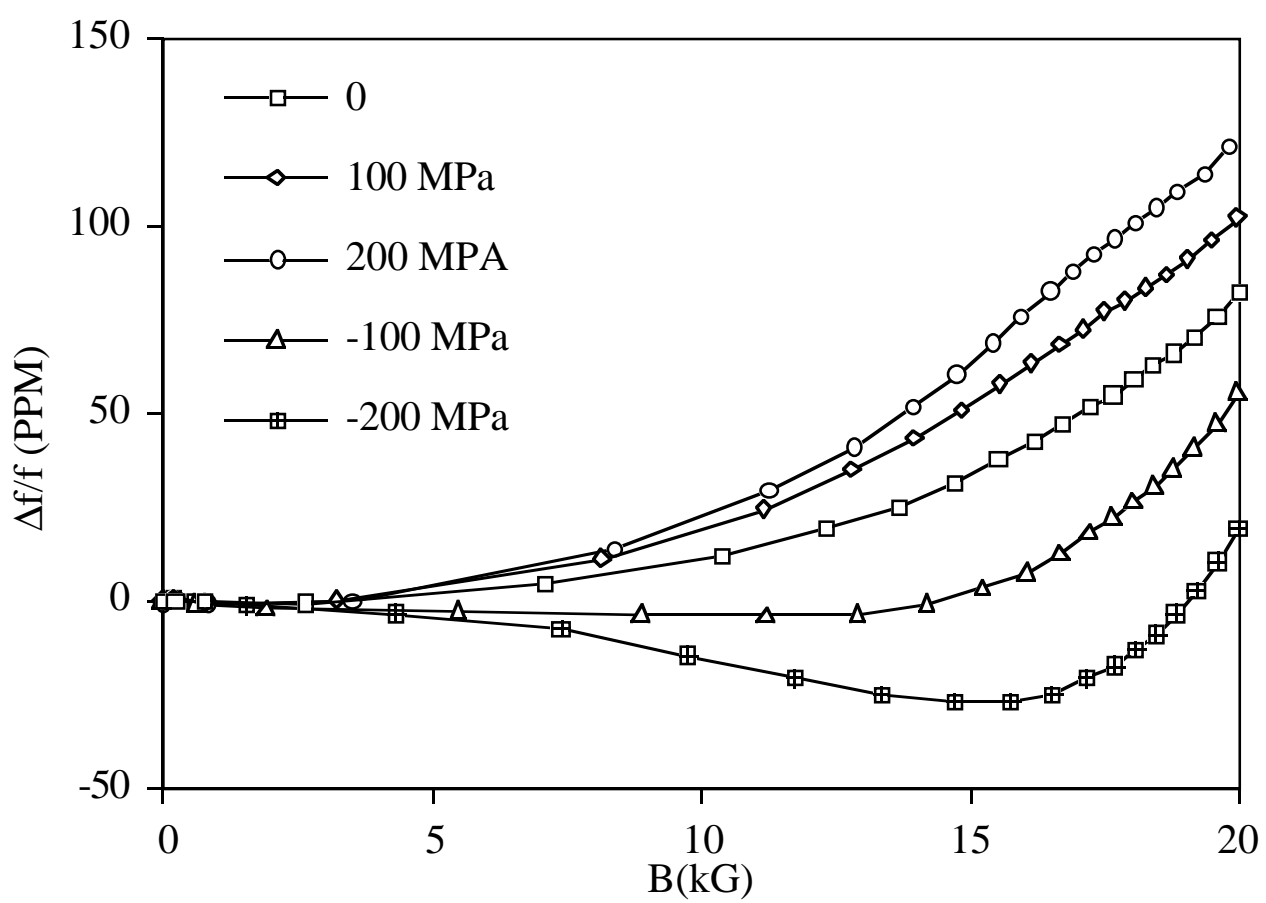

Fig. 3. MAC curves obtained at various applied stress levels with the U-class wheel steel sample on June 21, 1994.

the laboratory. The maximum $\Delta \mathrm{f}(\mathrm{T}) / \mathrm{f}$ fluctuation is +1 PPM per minute, which is too small to account for the irreproducibility shown in these figures. Hence, the variations in the $\Delta \mathrm{f}(\mathrm{B}) / \mathrm{f}$ curves are not solely due to temperature variations. They must also be due to some irreversible processes that occur in the samples due to the repeated changes in the stress state.

At certain selected levels of applied stress the measurements were repeated in a shorter time scale and compared with those included in the previous figures. Fig. 8 shows the results of four $\Delta \mathrm{f}(\mathrm{B}) / \mathrm{f}$ curves obtained with the U-class sample under -200 MPa while the room temperature in the laboratory was not fluctuating. These results suggest that the variations in $\Delta \mathrm{f}(\mathrm{B}) / \mathrm{f}$ observed over a long term (several days) may be worse than the short

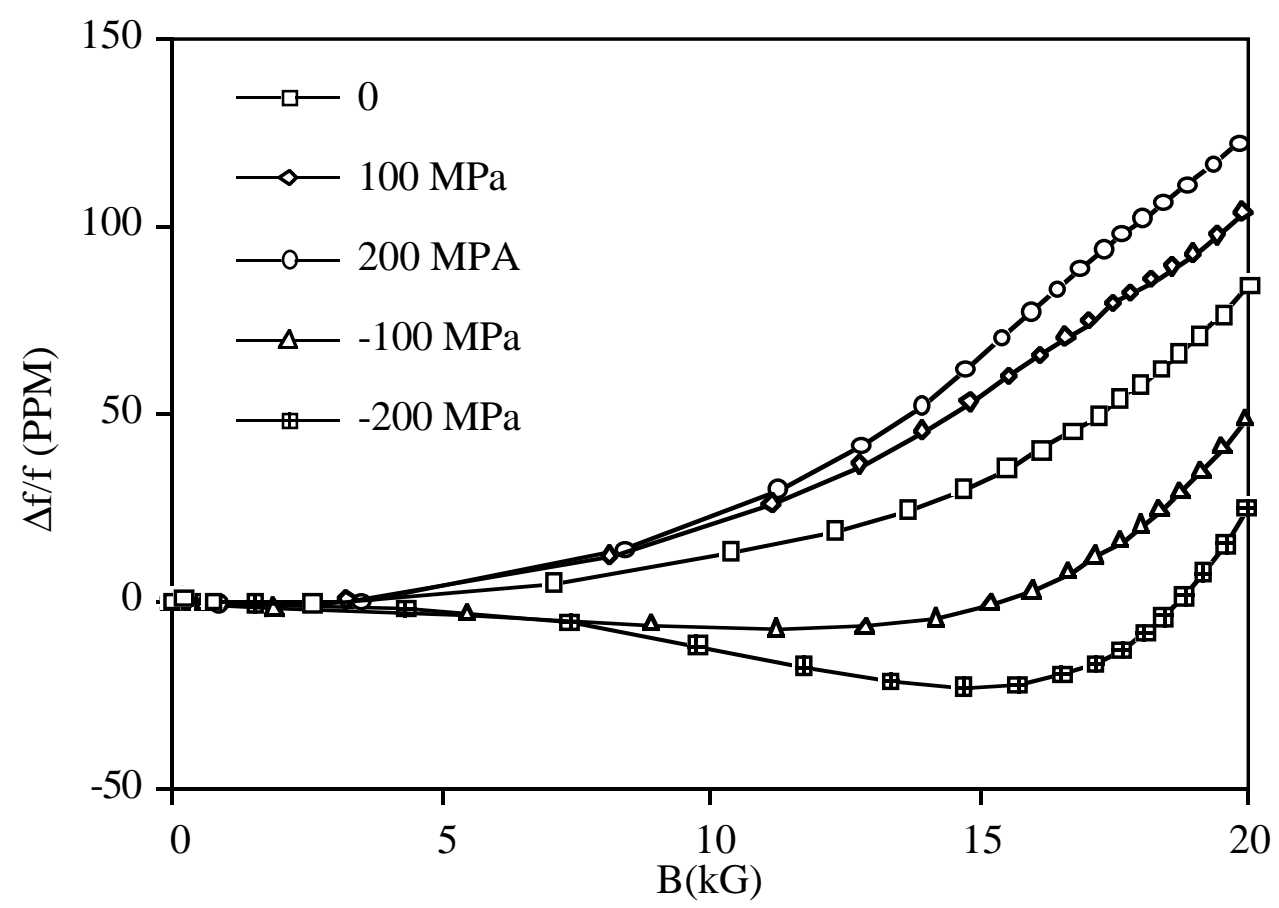

Fig. 4. Results of repeated measurements of Fig. 3 on June 24, 1994. 


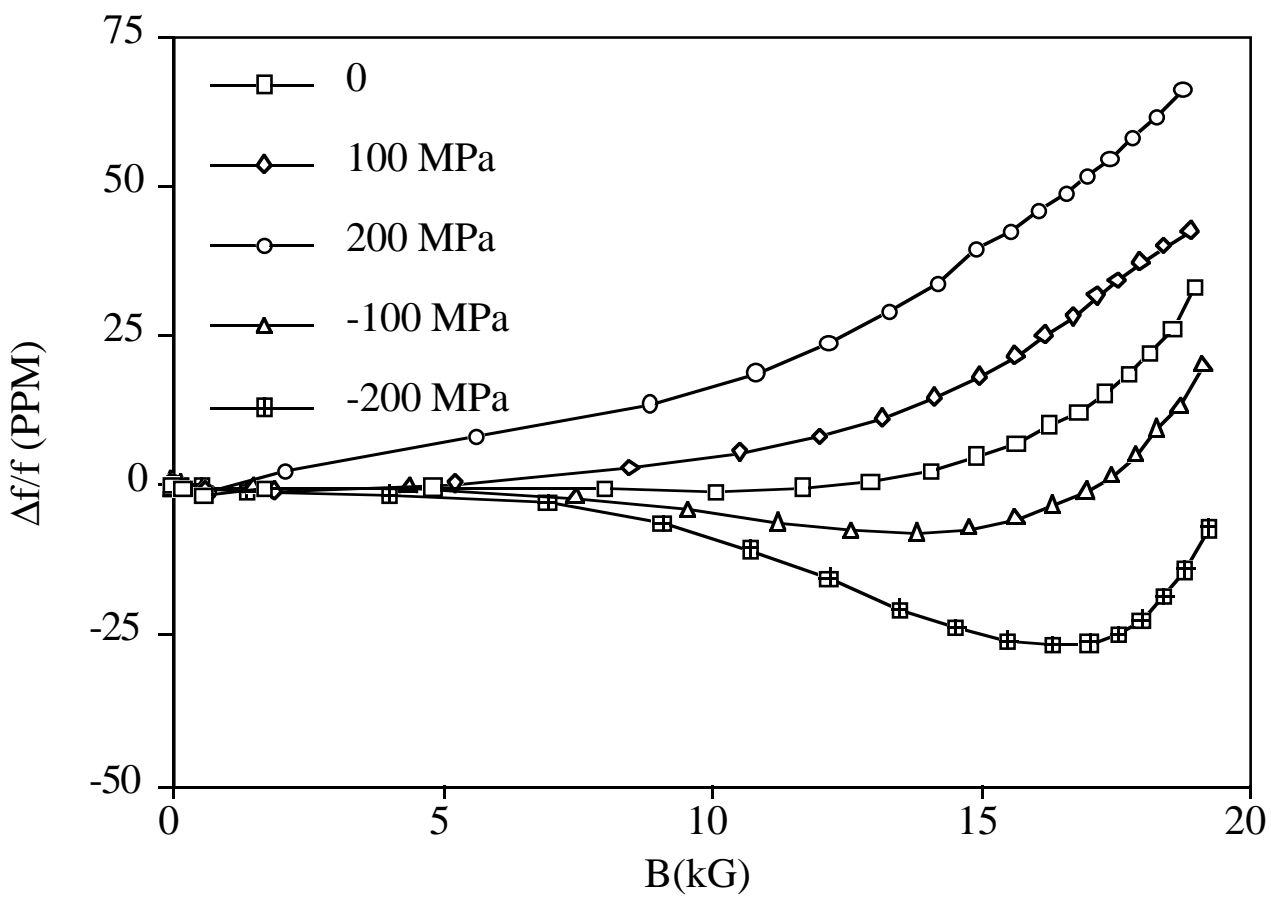

Fig. 5. MAC curves obtained at various applied stress levels with the C-class wheel steel sample on June 17, 1994.

term (several hours) variations. Fig. 9 shows the $\mathrm{B}-\mathrm{H}$ curves corresponding to the $\Delta \mathrm{f}(\mathrm{B}) / \mathrm{f}$ curves of Fig. 8. The results clearly show that the overall magnetic state is reproducible. The curves fall precisely on top of one another and it is only possible to identify one curve from the figure. Fig. 10 shows the time dependence of the C-class sample under a $200 \mathrm{MPa}$ load. Two of the curves were obtained less than three hours apart (indicated by circles and triangles) and are displayed along with the previous results. It is apparent that in this sample short term irreproducibility is much more pronounced. The B-H curves in this sample were as repeatable as that of the U-class sample.

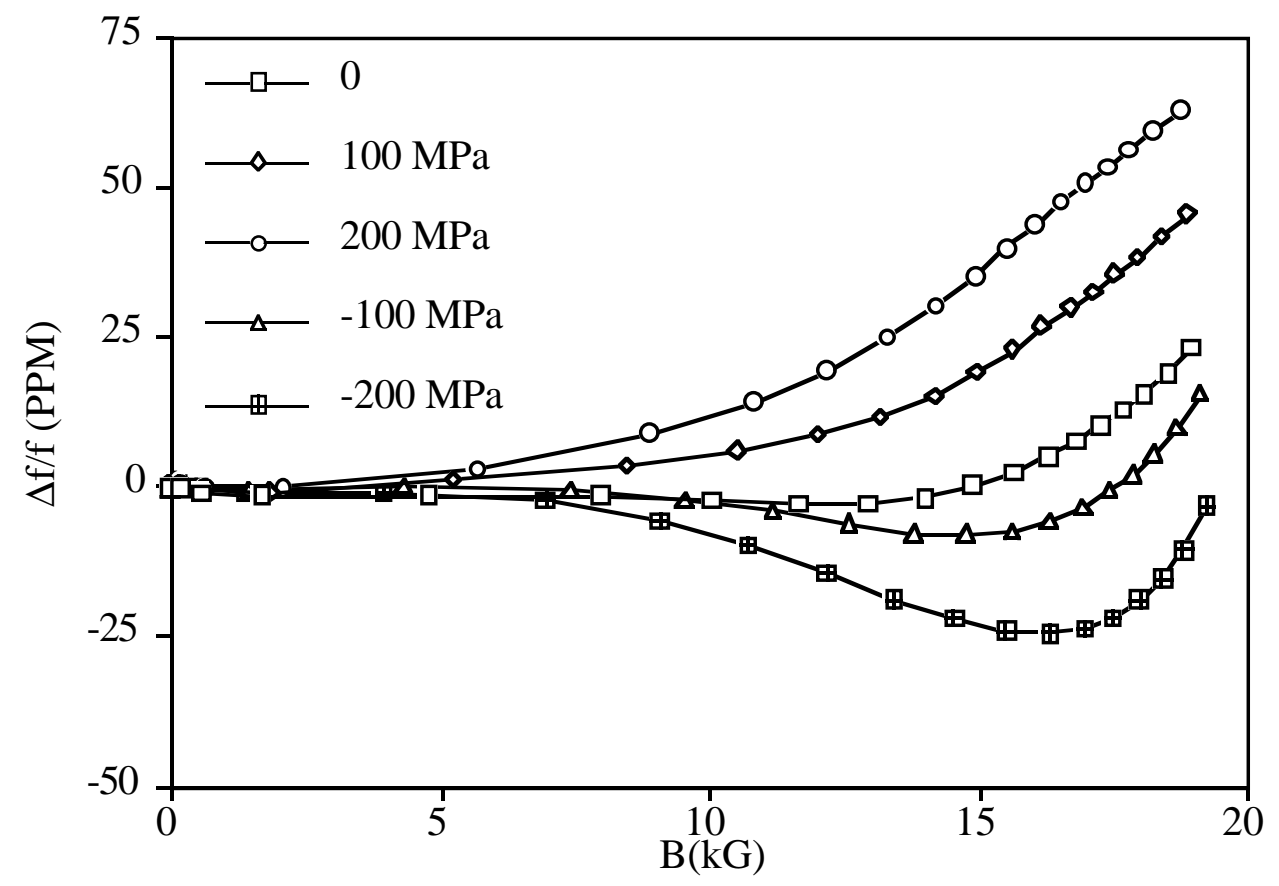

Fig. 6. Results of repeated measurements of Fig. 5 on June 23, 1994. 


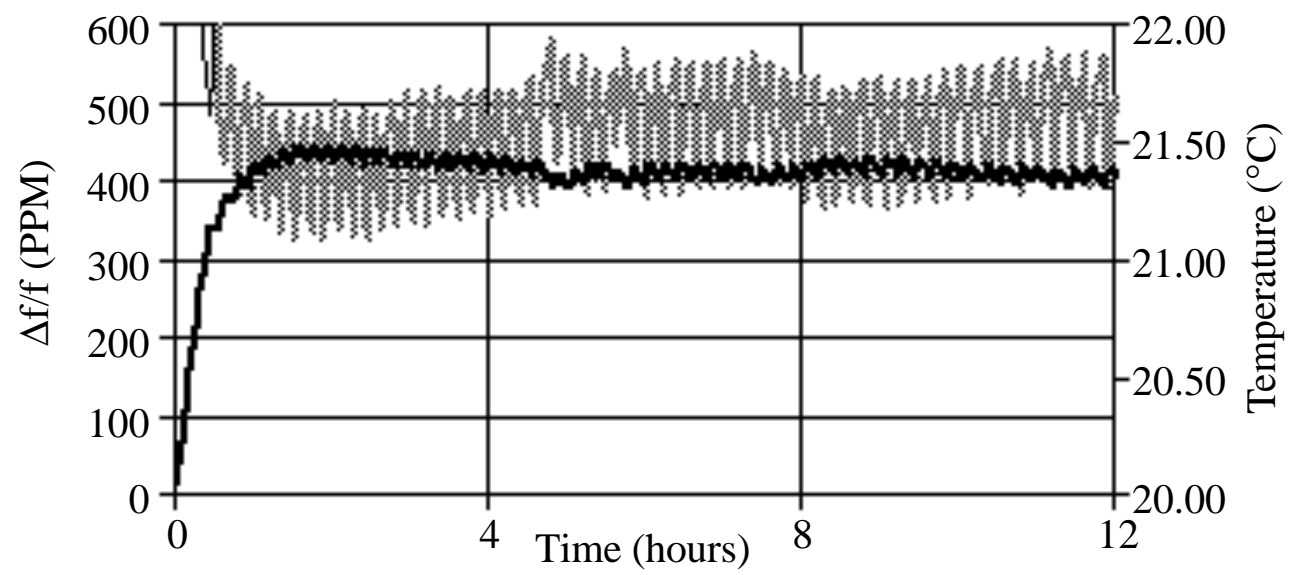

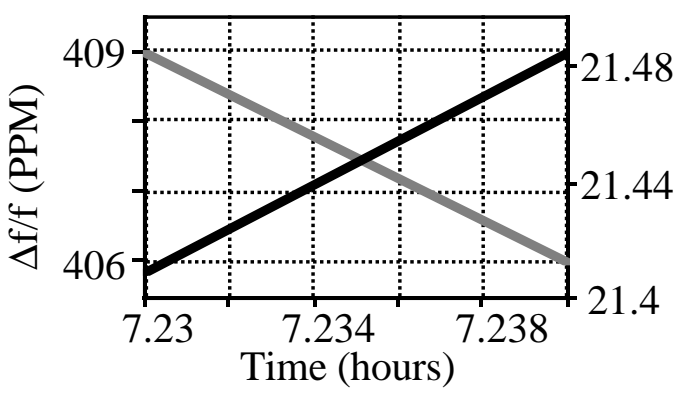

Decreasing Temperature

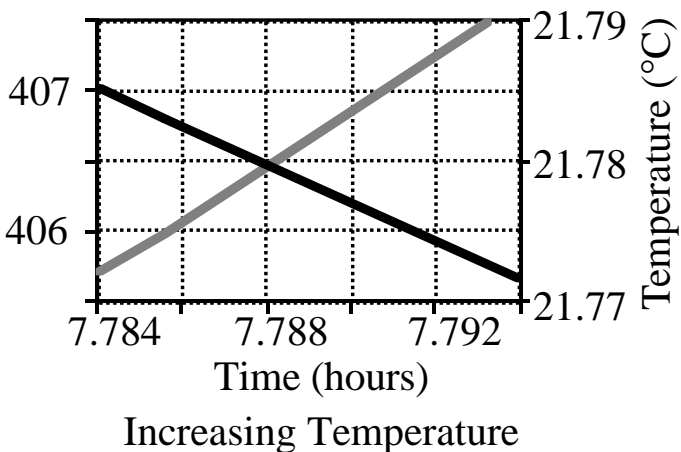

Increasing Temperature

Fig. 7. Periodic fluctuation of room temperature and $\Delta \mathrm{f} / \mathrm{f}$ observed over a period of 12 hours. The periodic temperature variation is due to the thermostat-controlled operation of the air-conditioner of the laboratory. The maximum rate of fluctutation of $\Delta \mathrm{f} / \mathrm{f}$ is $+1 \mathrm{PPM}$ per minute.

\section{DISCUSSION}

From the experimental results presented in the previous section it is clear that the irreproducibility in $\Delta \mathrm{f}(\mathrm{B}) / \mathrm{f}$ curves is not due to any undesirable artifact involved in the experimental procedures or any environmental changes. It is also clear that the variations are more

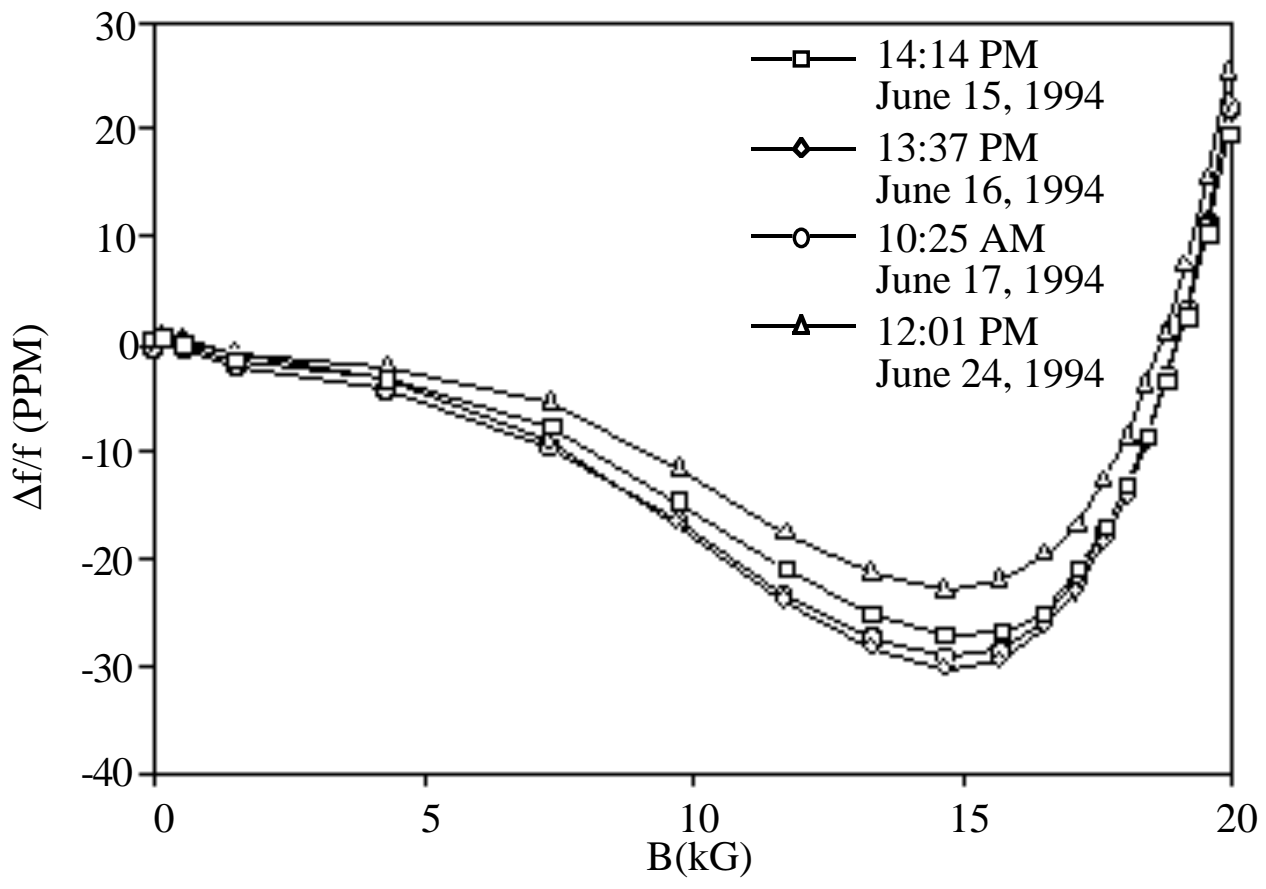

Fig. 8. MAC curves obtained with the U-class wheel steel sample under $-200 \mathrm{MPa}$ compared with the curves from Figs. 3 and 4. 


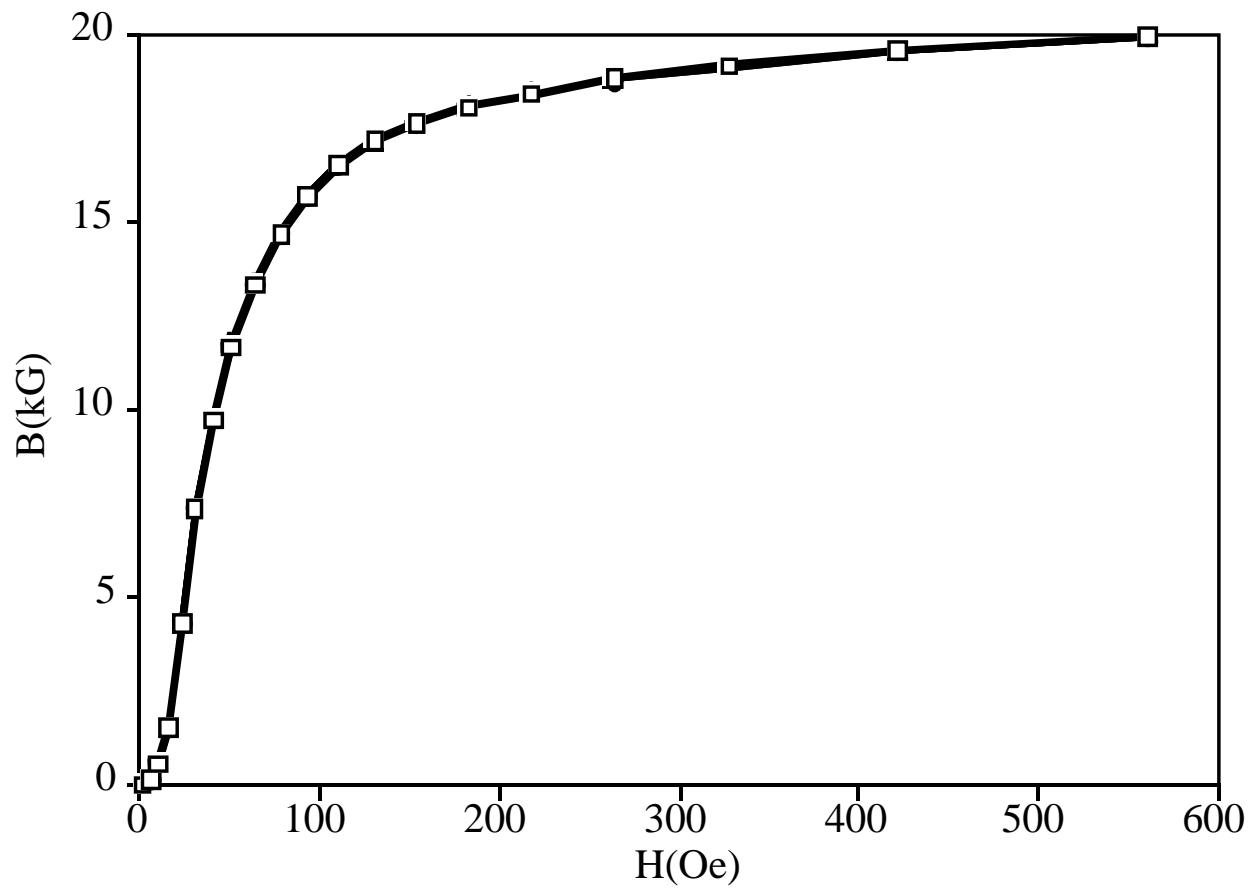

Fig. 9. B-H curves corresponding to the MAC curves of Fig. 8.

pronounced over the short term, i. e., several hours compared to that observed over a longer period of time, i. e., several days. Such a trend suggests that the irreproducibility probably is caused by a change in the material characteristics that has a relaxation time which is on the order of several days. At the same time, the involved process is not totally reversible. The only physical factor that can fit such a description is the uniaxial stress-induced motion of dislocations [6].

Under the applied uniaxial load cycles, the dislocations move through the sample, but due to various types of motion resisting mechanisms which are also stress and time dependent, their paths are not reproduced. Hence, after a given number of load cycles the system

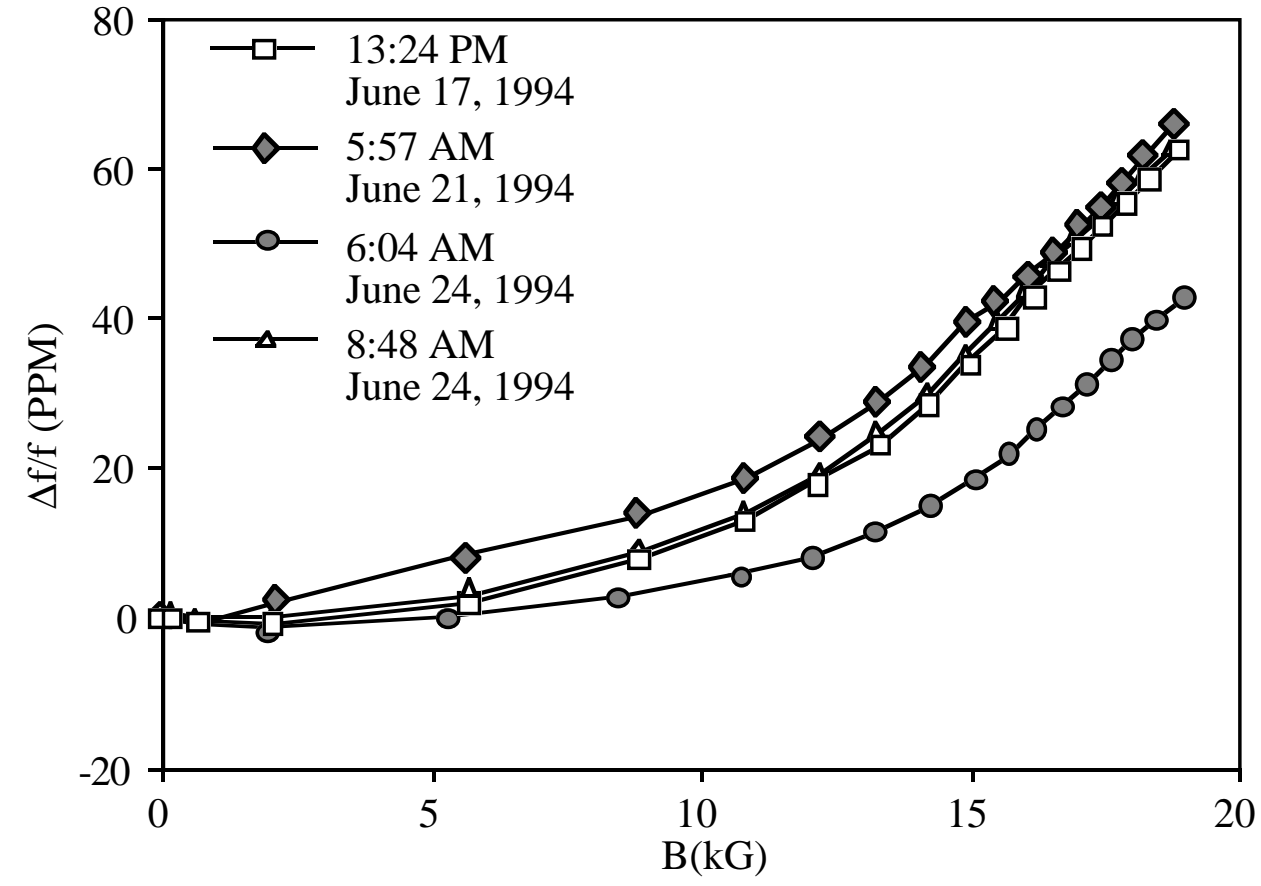

Fig. 10. MAC curves obtained with the C-class wheel steel sample under $200 \mathrm{MPa}$ compared with the curves from Figs. 5 and 6. 
of dislocation can be in a thermodynamically unstable state and it tends to restore its origi-

nal state through thermally-activated motion of dislocations [7]. At the same time, non- $180^{\circ}$ magnetic domain walls and dislocations interact through the lattice strain fields they create [8]. Therefore, any variation in the dislocation structure will affect the distribution of the applied magnetic field-induced motion of domain walls especially those none $180^{\circ}$ domain walls causing the MAC responses. Hence, from the experimentally observed short term variations of the $\Delta \mathrm{f}(\mathrm{B}) / \mathrm{f}$ curves which are more pronounced than that of the long term, one can argue that the origin of such irreproducibility is due to the motion of dislocations.

\section{SUMMARY}

A systematic experimental study was performed to identify the potential source of the irreproducibility in the MAC responses. The test results provided sufficient evidence to rule out any contribution from artifacts that can be involved in the experimental procedures. Based on the short and long term variations of the $\Delta f(B) / f$ curves, it was concluded that the irreproducibility is most probably due to the motion of uniaxial stress-induced dislocations.

\section{REFERENCES}

1. D. Utrata and M. Namkung, Presented at Review of Progress in Quantitative NDE, Brunswick, Maine, (July 30 - August 4, 1993).

2. M. Namkung and D. Utrata, in Review of Progress in Quantitative NDE Vol. 7B edited by D. O. Thompson and D. E. Chimenti, p. 1429 (Plenum Press, New York, 1987).

3. G. W. Ranthenau, in Magnetic Properties of Metals and Alloys, (American Society of Metals, Cleveland, Ohio, 1958).

4. M. Namkung, D. Utrata, W. T. Yost, P. W. Kushnick and J. L. Grainger, Proc. of IEEE Ultrasonic Symposium, Vol. 2, 1061 (1987).

5. S. Chikazumi, Physics of Magnetism, (John Wiley \& Sons, 1964).

6. C. S. Barrett, Structure of Metals, 2nd ed. (McGraw-Hill, New York, 1952).

7. G. B. Olsen and M. Cohen, Dislocations in Solids, Vol. 7, edited by F. R. N. Nabarro (North-Holland, New York, 1986).

8. H. Trauble, in Magnetism and Metallurgy, Vol. 2, edited by A. E. Berkowitz and E. Kneller (Academic Press, New York, 1969). 